\title{
Spin-injection terahertz radiation in magnetic junctions
}

\author{
S.G. Chigarev ${ }^{1}$, E.M. Epshtein ${ }^{1 *}$ Yu.V. Gulyaev ${ }^{1}$, I.V. Malikov², \\ G.M. Mikhailov ${ }^{2}$, A.I. Panas ${ }^{1}$, V.G. Shofman ${ }^{1}$, P.E. Zilberman ${ }^{1}$ \\ ${ }^{1}$ V.A. Kotelnikov Institute of Radio Engineering and Electronics \\ of the Russian Academy of Sciences, 141190 Fryazino, Russia \\ ${ }^{2}$ Institute of Microelectronics Technology and High Purity Materials \\ of the Russian Academy of Sciences, 142432 Chernogolovka, Russia
}

\begin{abstract}
Electromagnetic radiation of $1-10 \mathrm{THz}$ range has been found at room temperature in a structure with a point contact between a ferromagnetic rod and a thin ferromagnetic film under electric current of high enough density. The radiation is due to nonequilibrium spin injection between the structure components. By estimates, the injection can lead to inverted population of the spin subbands. The radiation power exceeds by orders of magnitude the thermal background (with the Joule heating taking into account) and follows the current without inertia.
\end{abstract}

\section{Experiment}

The structure in study is an inhomogeneous magnetic junction. It consists of two main ferromagnetic components: a hard magnetic rod of hardened steel and a soft magnetic (Permalloy) thin film of nanosize thickness (Fig. 1). To simplify realization and calculations, the whole structure has cylindrical form. The rod is sharpened at the end down to diameter of $2 R=10-100 \mu \mathrm{m}$, the film thickness is $h=10-100 \mathrm{~nm}$.

The structure described has an important property [1, 2], namely, because of the current continuity, high spin-polarized current density may be reached in the film exceeding by $2 R / h \gg 1$ times the current density in the rod. Then an energy minimum for majority electrons appears in the rod center (Fig. 1). Such electrons concentrate near the minimum. With the current increasing, the Fermi quasilevel $\varepsilon_{F \downarrow}$ rises and the electrons from the minimum tend to penetrate into the film outside the rod. At the distance $0<r-R<l$ from the rod edge smaller than the spin

*E-mail: epshtein36@mail.ru 


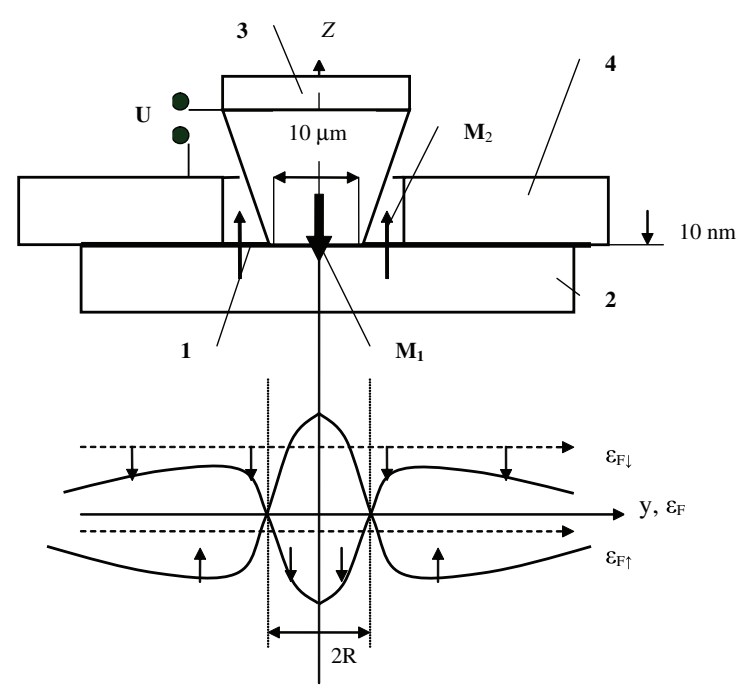

Figure 1: The structure in study consists of a Permalloy (Py) film (1), a substrate of oxidized silicon (2), a ferromagnetic rod (3), and a copper contact ring (4). Voltage $U$ is applied between the rod and the ring, so that direct current flows along the rod axis and through the Py film. The rod is magnetized along its axis. The magnetization vector $\mathbf{M}_{1}$ inside the rod is shown with the heavy arrow, the $\mathbf{M}_{2}$ component of the magnetization vector in the closing magnetic field outside the rod is shown with two heavy arrows. Note, that $\mathbf{M}_{2}$ depends on the properties of the magnetic circuit under the substrate. The coordinate system is shown at the bottom of the figure with $z$ axis coinciding with the rod axis. The bottoms of the spin subbands in the Py film are presented (qualitatively) with solid lines, the spin directions in the subbands are shown with thin arrows. The Fermi level $\varepsilon_{F}$ splits to two quasilevels $\varepsilon_{F \downarrow}$ and $\varepsilon_{F \downarrow}$ (dashed lines) in presence of the current. 


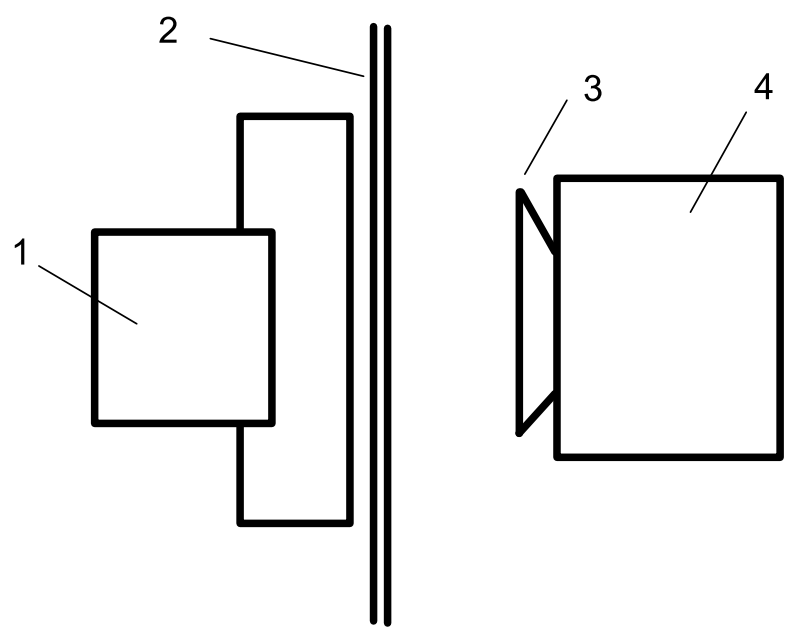

Figure 2: The scheme of the receiving unit consisting of a radiator (1), a low frequency filter (2), a high frequency filter (3), and Golay cell (4).

diffusion length $l \sim 20-30 \mathrm{~nm}$, the majority spins are directed opposite to $\mathbf{M}_{2}$, this means inverted spin population. The inversion is promoted by an energy maximum for the electrons with minority spins which appears near the rod axis (see Fig. 11). The energy maximum leads to withdrawal of such electrons from the rod. Thus, spin separation occurs in the junction because of its inhomogeneity, so that the electromagnetic radiation power may be gained under intersubband transitions.

To detect radiation, we placed the receiver on the substrate side within $L \geq 100 \mathrm{~mm}$ distance of the substrate, so that the receiver could rotate around the radiation source.

The problem of the current-driven spin population in ferromagnetic films and observing radiation under intersubband transitions evokes great interest for a long times. Theoretical estimates of the expected $\mathrm{THz}$ frequencies have been made in a number of works 3, 4, 5, 6. So, a Golay cell was used as a detector and two filters to fix the frequency range, namely, a metal grid with $125 \times 125 \mu \mathrm{m}$ for low frequencies and a standard TYDEX filter for high frequencies (Fig. 2). As a result, the studied frequency range was $1-10 \mathrm{THz}$. The receiver was in the wave zone, because $L \gg \lambda$ condition was fulfilled, $\lambda$ being the radiation wavelength. 


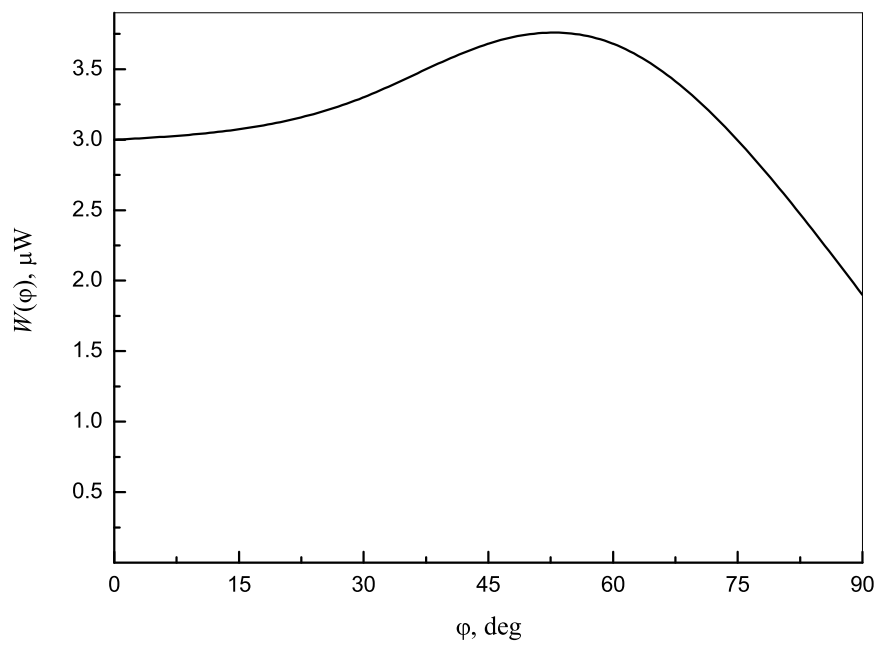

Figure 3: The angular dependence of the received radiation power $W(\varphi)$ at current about $\sim 400 \mathrm{~mA}$.

\section{The measurement results and discus- sion}

Let us start with measuring dependence of the radiation intensity on the observation angle $\varphi$, i.e., on the angle between the rod axis and the normal to the objective plane. A typical angular dependence is shown in Fig. 3 . It is seen that the radiation has no pronounced directivity, so that an average power $\bar{W} \sim 2.5 \mu \mathrm{W}$ falls on the objective at any angle. With the objective diameter of $\sim 6 \mathrm{~mm}$, this gives the total radiation power of $W_{\text {total }} \sim 10 \mathrm{~mW}$ to the complete solid angle $4 \pi$. It is interesting to estimate the effective absolute temperature $T$ of a body that radiates such a power. In the frequency range in study, the Rayleigh-Jeans law is fulfilled,

$$
W_{\text {total }}=\frac{2 \pi k T S}{c^{2}} \int_{f_{1}}^{f_{2}} f^{2} d f,
$$

where $k$ is the Boltzmann constant, $c$ is the light velocity, $S$ is the area of the radiating surface, $f$ is the radiation frequency. With $S \sim 0.1 \mathrm{~cm}^{2}$ and $f_{1} \sim 10^{12} \mathrm{~Hz}, f_{2} \sim 10^{13} \mathrm{~Hz}$, we have $T \geq 3000 \mathrm{~K}$. Such high temperatures were absent in the experiment. Hence, the power of the receiving radiation is too high to be explained in terms of the radiator heating.

At the next stage, the receiving power has been measured as a function of the current at $\varphi=0$. The results are shown in Fig. [4 The radiation appears starting with some threshold current. Under decreasing the current, the backward branch of the $W(\varphi)$ dependence does not coincide with the 


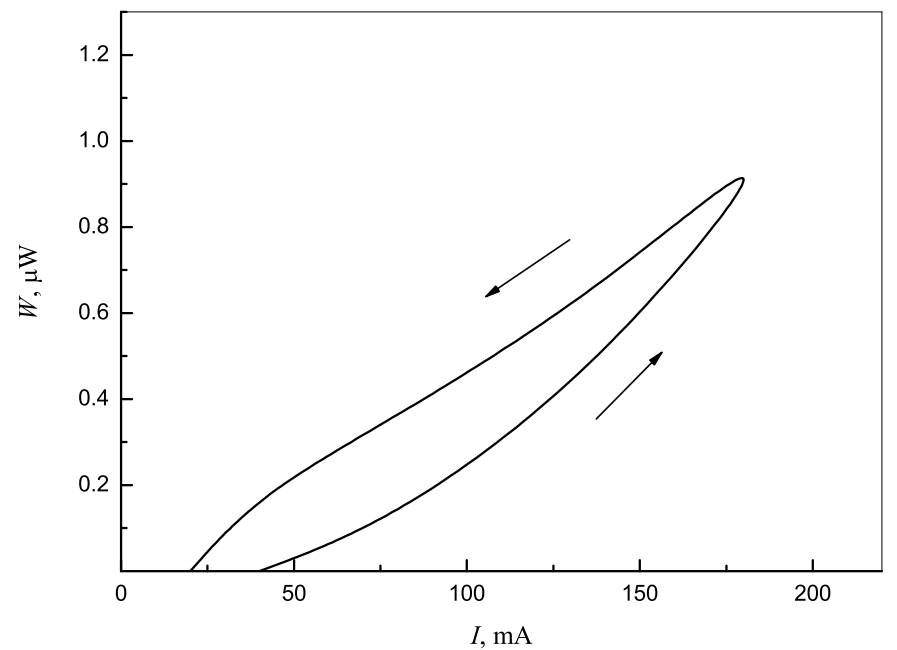

Figure 4: The radiation power as a function of the current. The arrows indicate direction of the current change.

forward one, so that a loop appears. It may be assumed that the sample properties change under current increasing, so that we have somewhat another medium during the current decreasing. Such a behavior is related with the Permalloy film coercivity. Indeed, the magnetic flux from the rod reverses the film magnetization. The injected conduction electrons contributes to this reversal, too. Under current decreasing, the injection contribution decreases, but the film state follows the current with some delay. Note, that the threshold existence is an additional indication to non-thermal nature of the radiation observed.

It is important to know, how the radiation follows the current under the latter changing in time, with jumps and turning off. It is seen from Fig. 5 that the radiation tracks all the variations of the current. The only smooth character of the radiation pulse fronts under abrupt jumps of the current and a small smooth "tail" near zero may be related with thermal effects. Such a suppression of the thermal radiation is due to using substrates with high thermal conductivity. Early 1, 2, 8, a fluoroplastic layer was used in the substrate that slowed down the heat removal and led to intense heating the sample by the current, so that the thermal radiation increased, while the noh-thermal one suppressed. In present investigation, the sample temperature remains low enough (no more than $60-100^{\circ} \mathrm{C}$ ), so that the radiation of magnetic nature exceeding substantially the thermal background becomes the main effect.

The spin-polarized current injects spin and disturbs spin equilibrium near the contact between the junction layers (see review [9]). According the estimates in Ref. [8], this can lead to inversion of the spin subband 


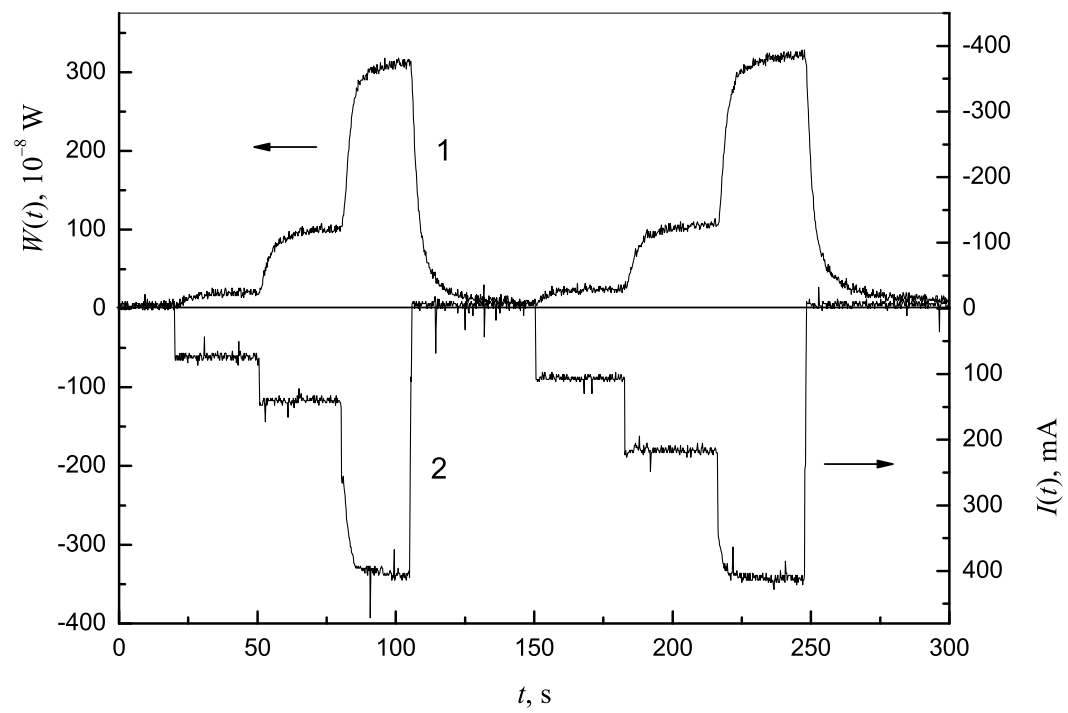

Figure 5: The radiation power $W(t)$ (curve 1, the left ordinate axis) and current (curve 2, the right ordinate axis) as functions of time.

population. We show below that such an inversion really takes place in our structure (Fig. 1) under high currents because of opposite directions of $\mathbf{M}_{1}$ and $\mathbf{M}_{2}$ magnetizations in the forward and closing magnetic fluxes. The following equation is solved for the conduction electron spin polarization $\mathbf{P}$ in the film [8]:

$$
\frac{d^{2} \mathbf{P}}{d r^{2}}+(1-2 \nu) \frac{1}{r} \frac{d \mathbf{P}}{d r}-\frac{\mathbf{P}-\overline{\mathbf{P}}}{l^{2}}=0,
$$

where $r$ is the distance from the rod axis, $\overline{\mathbf{P}}$ is the equilbrium spin polarization,

$$
\nu=\frac{R}{2 l} \frac{j}{j_{D}},
$$

$j$ is the current density in the $\operatorname{rod}, j_{D}=e n l / \tau$ is a characteristic current density $\left(j_{D} \sim 10^{9} \mathrm{~A} / \mathrm{cm}^{2}\right), e$ is the electron charge, $n$ is the electron density in the film, $\tau$ is the spin relaxation time. Equation (2) is solved under condition that the polarization is equilibrium ah large distance from the rod, i.e., $\mathbf{P}=\overline{\mathbf{P}}(\infty)$, while the spin current is continuous at rod boundary $r=R$. Then we have at $r=R$ [8]

$$
\delta \mathbf{R}=\left(Q_{1} \hat{\mathbf{z}}\left(\hat{\mathbf{M}}_{1} \cdot \hat{\mathbf{M}}_{2}\right)-\overline{\mathbf{P}}\right) \frac{j(R)}{j_{D}} \frac{K_{\nu}(R / l)}{K_{\nu+1}(R / l)},
$$

where $\delta \mathbf{P} \equiv \mathbf{P}-\overline{\mathbf{P}}$ is the nonequilibrium part of the spin polarization, $\hat{\mathbf{z}}$ is the unit vector along the rod axis, $Q_{1}=\left|\sigma_{\downarrow}-\sigma_{\uparrow}\right| /\left(\sigma_{\downarrow}+\sigma_{\uparrow}\right)$ is the spin 
polarization of the rod conductivity, $\sigma_{\downarrow}$ and $\sigma_{\uparrow}$ are the partial conductivities of the electrons with spin parallel and antiparallel to $\hat{\mathbf{M}}_{1}$ vector, respectively, $\hat{\mathbf{M}}_{1,2}=\mathbf{M}_{1,2} /\left|M_{1,2}\right|, K_{\nu}$ is the modified Bessel function of the second kind. Because of $\left(\hat{\mathbf{M}}_{1} \cdot \hat{\mathbf{M}}_{2}(R)\right)<0$, it follows from Eq. (4) that inverse population can be reached, $\mathbf{P}(R)=-\hat{\mathbf{z}}|\mathbf{P}(R)|$ at high enough $j / j_{D}$ ratio. The estimates in Ref. 8 show that the inversion is reached at $j \sim 10^{8}-10^{9} \mathrm{~A} / \mathrm{cm}^{2}$.

Note, that the dependence on the current in Eq. (3) appears not only explicitly, but via the $\nu$ index of the Bessel function also. Because of that fact, the $|P(R)|$ polarization and the radiation power can change substantially (by more than 30\%). Such change may not be explained as thermoelectric effects (Nernst-Ettingshausen effect and so on) which do not exceed fractions of a percent in metals. The observed substantial "nonreciprocity" of the radiation may be additional argument in favor of the spin injection mechanism.

Let us mention some check experiments which confirm magnetic nature of the observed $\mathrm{THz}$ radiation. First, the non-thermal radiation was disappeared totally, when the steel rod was replaced with a nonmagnetic (copper) one, the intensity was decreased, and nonreciprocity was not observed. Second, non-thermal radiation and nonreciprocity were disappeared also under replacing the Permalloy film with molybdenum one, the steel rod being retained. Third, attaching a core of soft magnetic steel made it possible to carry the closing magnetic flux off the sample in study. As a result, $\hat{\mathbf{M}}_{2}$ component was decreased down to zero. This manifested in experiment as disappearance of $\mathrm{THz}$ radiation following the current, and increasing in time thermal radiation due to nonradiative relaxation of the spin polarized along $z$ axis.

\section{Summary}

We have observed rather intense radiation from a magnetic junction within 1-10 $\mathrm{THz}$ range. The radiation power exceeds the thermal background by an order of magnitude. The radiation has no pronounced directivity, its total power is about $\geq 10 \mathrm{~mW}$. The radiation appears with a current exceeding some threshold. The radiation power rises with the current. Under decreasing current, the radiation power falls and disappears at another threshold current. The current polarity influence the radiation intensity changing it by more than $30 \%$.

As estimates show, an intense nonequilibrium spin injection occurs near the contact in the structure under current flow. It is possible that domains with inverse population of the spin subbands can appear. The spin injection generates radiation with nonreciprocity relative to the current polarity.

The authors are grateful to Yu.G. Kusraev, O.V. Betskii, G.A. Ovsyannikov and Yu.G. Yaremenko for useful discussions.

The work is supported by the Russian Foundation for Basic Research, Grant No. 02-00030. 


\section{References}

[1] S. G. Chigarev, A. I. Krikunov, P. E. Zilberman, A. I. Panas, E. M. Epshtein, J. Commun. Technol. Electron. 54, 708 (2009).

[2] Yu. V. Gulyaev, P.E. Zilberman, A.I. Panas, S. G. Chigarev, E. M. Epshtein, J. Commun. Technol. Electron. 55, 668 (2010).

[3] A. M. Kadigrobov, Z. Ivanov, T. Claeson, R. I. Shekhter, M. Jonson, Europhys. Lett. 67, 948 (2004).

[4] A. M. Kadigrobov, R. I. Shekhter, M. Jonson, Phys. Low Temp. 31, 463 (2005).

[5] Yu. V. Gulyaev, P. E. Zilberman, A. I. Krikunov, A. I. Panas, E. M. Epshtein, JETP Lett. 85, 160 (2007).

[6] Yu. V. Gulyaev, P.E. Zilberman, E. M. Epshtein, A. I. Panas, A. I. Krikunov, RF Patent No. 2344528 (2009).

[7] L. D. Landau, E. M. Lifshitz, Statistical Physics (Pergamon, Oxford, 1980).

[8] Yu. V. Gulayev, P.E. Zilberman, S. G. Chigarev, E. M. Epshtein, J. Commun. Technol. Electron. 55, 1132 (2010).

[9] Yu. V. Gulyaev, P.E. Zilberman, A.I. Panas, E. M. Epshtein, Physics - Uspekhi 52, 335 (2009). 